\title{
Expertengespräch: Dermatologie
}

Das Arsenal der Schulmedizin ist bei Hautkrankheiten rasch erschöpft. Über die Methoden des Einsatzes der Chinesischen Medizin und der Homöopathie geben Dr. med. Hoa Thanh Huynh und Dr. med. Beatrix Gessner im Expertengespräch Auskunft.

Eignen sich die Methoden der ganzheitlichen Medizin für die Behandlung von Hautkrankheiten?

Thanh Huynh: Absolut! Dabei spielt die Chinesische Medizin sicherlich eine hervorragende Rolle, denn sie blickt auf eine Tradition zurück, die bei der Behandlung von Hauterkrankungen jahrhundertealt ist. Es sind nicht nur die Erfahrung und die Vergangenheit, die die Chinesische Medizin dazu prädestinieren, sondern auch die Gegenwart. Heute gibt es in China grosse Kliniken für Dermatologie, in denen Hauterkrankungen mit der chinesischen Arzneimitteltherapie behandelt werden.

Gessner: Aus der Sicht der Homöopathie auf alle Fälle. Ich arbeite jetzt seit 20 Jahren in einer homöopathisch konzentrierten Praxis und habe sehr viele Hautpatienten. Heute kommen etwa $40 \%$ meiner Patienten mit Hautproblemen zu mir. Die ganzheitlichen Methoden eignen sich unbedingt zur Behandlung - und sie lohnen sich. Das ist für mich auch ein Grund gewesen, in einer schulmedizinischen Hautklinik aufzuhören und mich auf die Homöopathie zu konzentrieren. Ich habe gemerkt, dass viele dermatologische Medikamente gravierende Nebenwirkungen mit sich bringen und das Spektrum der schulmedizinischen Methoden relativ eng ist. Als Schulmediziner hat man die Möglichkeit der Externa, der äußeren Lokalbehandlung. Da kann man mit
Leichterem beginnen, beispielsweise mit der Pflege und dem Einfetten. Wenn es um entzündliche Hautkrankheiten geht, stehen immer nur Kortison oder ein Ersatzpräparat auf dem Programm. Wenn es um gravierende Probleme geht, gibt es Kortison in Tablettenform, und bei Entzündungen, die sich auf der Haut bilden und eitrig werden, verordnet man antibiotische Salben. Aber viel mehr wird in der Schulmedizin nicht geboten. Die Homöopathie bietet eine grössere therapeutische Vielfalt und ist schonender.

\section{Mit welchen Problemen kom- men Patienten zu Ihnen in die Behandlung?}

Gessner: Häufig sind Allergien, Ekzeme, Neurodermitis, Schuppenflechte und Akne. Ich habe viele Patienten, die sich eine schonende Behandlung wünschen. Besonders Eltern legen für ihre Kinder darauf Wert, dass man möglichst ohne Nebenwirkungen behandelt. Auch viele Erwachsene wünschen sich weniger gravierende Methoden. Aus meiner Sicht ist das vernünftig, denn mit der Haut passiert einiges, wenn man zum Beispiel immer Kortison aufträgt, um ein Ekzem einzudämmen. Zum einen kann es stärker wiederkommen, wenn man das Kortison absetzt. Das sehe ich häufiger. Es kann aber auch sein, dass sich das Ekzem in eine andere Schicht verschiebt. Die Haut bleibt dann zwar ruhig und das Ekzem ist verschwun- den. Dafür können dann aber andere Störungen auftreten. Zum Beispiel im Bronchialbereich, wie beispielsweise Asthma, oder im Darmbereich, wie etwa eine Darmentzündung oder zumindest ein vegetativ empfindlicher Darm. Das Krankheitsbild verschiebt sich weg von der Hauterkrankung. Es wandert von aussen nach innen, wie wir in der Homöopathie sagen.

Thanh Huynh: Zu mir kommen Patienten vor allem mit Psoriasis. Ich habe mich auf die Behandlung der Schuppenflechte spezialisiert. Viele Patienten kommen aber auch mit allen Sorten von Ekzemen zu mir. In meiner Praxis sehe ich zum Beispiel Neurodermitis, nummuläres Ekzem, dyshidrotisches Ekzem oder Kontaktekzeme. Gute Erfahrungen habe ich bei der Behandlung mit den Methoden der Chinesischen Medizin bei Babys oder bei Kindern gemacht, die unter Neurodermitis leiden. Sie sprechen sehr gut auf die chinesische Therapie an. Nach meiner Erfahrung gehören Akne, Rosazea und seborrhoische Dermatitis ebenfalls zu den sehr gut behandelbaren Erkrankungen. Auch Patienten mit übermässigem Schwitzen oder solche, die unter Juckreiz leiden, profitieren ebenfalls deutlich von der chinesischen Therapie. Ich habe aber auch einige Patienten mit Erkrankung der Pigmentierung, wie die Vitiligo oder Chloasma, die ich mit der Chinesischen Medizin behandle.

\section{KARGER}

Fax +49761 4520714 


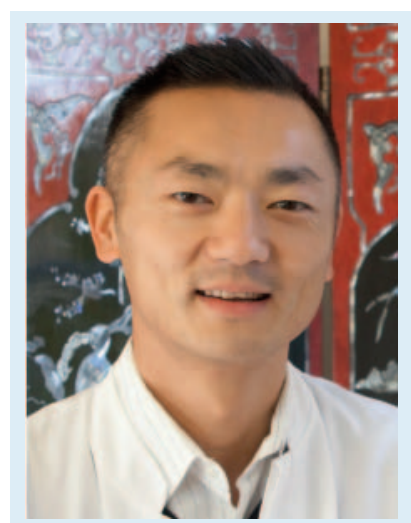

Dr. med. Hoa Thanh Huynh

(tan.huynh@see-spital.ch) ist Facharzt für Innere Medizin FMH mit Fähigkeitsausweis TCM/Akupunktur ASA. Sein Studium der Chinesischen Medizin absolvierte er bei der SMS/SAGA (München/Zürich). Die Vertiefung in dieses Fachgebiet und ein intensives Training in Akupunktur und Moxibustion brachte ihn nach Shanghai an die University of TCM (WHO-zertifiziert) und in angegliederte chinesische Spitäler. Ferner absolvierte er ein zweijähriges Curriculum in chinesischer Arzneimitteltherapie bei der SMS in München. In einem Nachdiplomstudium widmete er sich der chinesischen Dermatologie beim renommierten Arzt und Lehrer für Chinesische Medizin/Dermatologie Mazin Al-Khafaji in London. Dr. Thanh Huynh ist Gründer und Leiter des Zentrums für Chinesische Medizin im Spital Zimmerberg, Horgen. Dort führt er neben ambulanten Sprechstunden auch stationäre Konsilien in Chinesischer Medizin durch.

«Durch Harmonisieren und Aufbau kann das gute Resultat stabilisiert werden, sodass die Therapie nach 3-6 Monaten ausgeschlichen werden kann.»

Welche Rolle spielt die Psyche bei der Entstehung von Hautkrankheiten?

Thanh Huynh: Die Psyche kann bei Hauterkrankungen eine Rolle spielen; sie muss es nach dem Verständnis der Chinesischen Medizin aber nicht. Bei einem Patient mit Schuppenflechte kann sich die Haut unter Stress sicherlich verschlechtern. Aber es gibt auch die Rückwirkung von der Hauterkrankung auf die Psyche. Bei einer schwierig zu behandelnden Hauterkrankung leidet die psychische Verfassung des Patienten auch mit. Ich gehe bei meinem chinesischen $\mathrm{Be}$ handlungsansatz primär die Haut an und beobachte, dass sich die Psyche in den meisten Fällen verbessert, wenn die Haut geheilt ist.

Gessner: Nach meinem Verständnis spielt die Psyche bei Hauterkrankungen eine grosse Rolle. Für die Behandlung ist das sehr wichtig, weil wir in der Homöopathie sehr viel Wert auf die ganzheitliche Betrachtung des Menschen legen. Für die Wahl unserer Arznei ist es wichtig, seinen psychischen Zustand $\mathrm{zu}$ berücksichtigen und dann auch in die Behandlung einzubeziehen. Es ist wichtig zu wissen, ob der Mensch in schwierigen Umständen steckt oder in der Vergangenheit Krisen in seinem Leben durchgemacht hat. Deshalb nehme ich mir für ein Erstgespräch mit dem Patienten auch fast anderthalb Stunden Zeit.

\section{Was sind die Grundlagen Ihrer Behandlungsmethode bei der Haut?}

Thanh Huynh: In der Chinesischen Medizin gilt das Prinzip «Klären Harmonisieren - Aufbau». Durch Harmonisieren und Aufbau kann das gute Resultat stabilisiert werden, sodass die Therapie nach 3-6 Monaten ausgeschlichen werden kann.

Gessner: Homöopathie bedeutet, Gleiches mit Gleichem zu behandeln. Wir geben Arzneimittel, die in der Regel aus dem pflanzlichen oder mineralischen Bereich sind. Diese werden homöopathisch hergestellt, d.h. verdünnt und in sehr besonderen Schrittfolgen verschüttelt. Diese Medikamente sind im Grunde genommen energetische Medizin. Wenn wir jetzt die Behandlung eines Menschen beginnen, ist eine sorgfältige Anamnese entscheidend. Nehmen wir einmal den Fall eines Kinds mit Neu- rodermitis an. Es ist eineinhalb Jahre alt, und seit Monaten hat es Ekzeme, erst im Gesicht, jetzt am Körper; es kratzt sich ständig. Man hat schon versucht, gewisse Nahrungsmittel wegzulassen. Erst gab es eine leichte Besserung, jetzt wird es wieder schlimmer. Wenn die Familie kommt, schaut man sich natürlich die Haut an. Man guckt, wie das äußere Bild ist. Man fragt dann auch vor allen Dingen nach; wir nennen es Konstitutionstherapie: Wie war der ganze Werdegang des Menschen? Wie war die Schwangerschaft? Wie war die Geburt? Wie hat sich das Kind entwickelt? Wie geht es mit dem Essen? Was gibt es für Vorlieben und Abneigungen? Wie schläft das Kind? Wacht es zu bestimmten Zeiten auf? Verändert sich der Zustand bei Wärme oder Kälte, an der Sonne oder Luft? Als homöopathische Ärztin mache ich mir ein möglichst gutes Bild der Konstitution. Wenn man einen Erwachsenen behandelt, macht man sich ebenfalls ein Gesamtbild. Mit der Homöopathie können wir bei etwa $80 \%$ der Patienten eine Verbesserung feststellen.

\section{Wo geraten Sie mit Ihren Methoden der Ganzheitsmedizin an Ihre Grenzen?}

Thanh Huynh: Ganz klar bei Hauttumoren. Dort muss chirurgisch vorgegangen werden.

Gessner: Beim Hautkrebs behandle ich nicht homöopathisch. In diesen Fällen muss operiert werden. Wenn jemand einen sehr starken Hautschub hat, bewährt sich dann oft eine Ernährungstherapie. Oder in ganz besonders dramatischen Phasen, wie zum Beispiel bei Ekzemschüben bei Neurodermitis, bringt das Heilfasten oft einen Erfolg. Das Heilfasten bringt in kurzer Zeit eine deutliche Besserung des Juckreizes. 


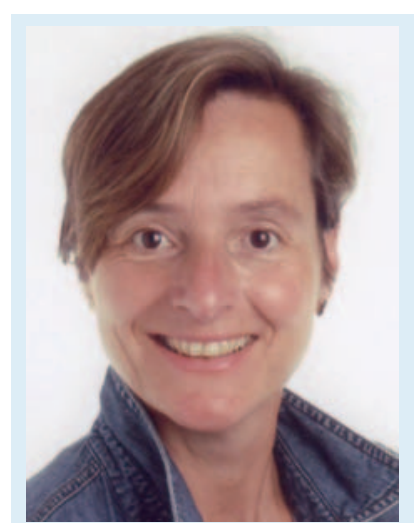

Dr. med. Beatrix Gessner

(dr.beatrixgessner@t-online.de) ist Ärztin für Homöopathie und Naturheilverfahren in Konstanz. Sie betreibt eine Privatpraxis und hat sich auf die Behandlung von Patienten mit Hautproblemen spezialisiert. Dr. Gessner hat an verschiedenen schulmedizinischen Hautkliniken gearbeitet, bevor sie sich der Homöopathie zugewandt hat. Sie hat eine Übersichtsarbeit über den Einsatz der Homöopathie in der Dermatologie veröffentlicht: «Die homöopathische Behandlung von Hauterkrankungen» (Z Klass Homöopathie 2005;52: 180-187).

«Homöopathie bedeutet, Gleiches mit Gleichemzubehandeln, aberwirberücksichtigen darüber hinaus die Befindlichkeit und die Probleme des Patienten.»

Kombinieren Sie Ihre Methode auch mit der Schulmedizin?

Thanh Huynh: Nicht oft, aber zum Beispiel bei einer bakteriellen Infektion oder bei einem Pilzbefall eines chronischen Ekzems habe ich auch schon ein Antibiotikum oder ein Pilzmittel eingesetzt. Ich bin auch westlicher Mediziner und habe keine Mühe damit, westliche Medikamente einzusetzen, wenn der Patient deutlich davon profitiert und keine $\mathrm{zu}$ grossen Nebenwirkungen davonträgt. Auf je- den Fall muss bei einem Notfall, zum Beispiel bei einem Asthmaanfall, pragmatisch und richtig reagiert werden. Da setze ich auch hoch dosiert Kortison ein.

Gessner: Gerade bei Hautkrankheiten beissen sich der homöopathische und der schulmedizinische Ansatz. Wenn bei einem Patienten Kortison verwendet wird, ist es sehr schwierig, gleichzeitig homöopathisch zu behandeln. Manchmal geht es aber nicht anders, und man muss dann auch beide Ansätze gleichzeitig verfolgen, um mit der homöopathischen Therapie einen Fuss in die Tür zu bekommen. Beim Asthma ist es beispielsweise ja sinnvoll, dass die Leute ihre Sprays weiterhin nehmen. Da sage ich nie, dass sie sofort die Sprays absetzen sollen, sondern fange mit einem homöopathischen Mittel an. Wenn ich sehe, dass sich der Zustand des Patienten bessert, kann man das Medikament leichter ausschleichen lassen.

\section{Behandeln Sie Hautkrankheiten bei Kindern anders als bei Erwachsenen?}

Thanh Huynh: Kinder sind sehr sensibel und reagieren oft schon sehr gut auf lokale Kräutertherapien. Deshalb setze ich diese zuerst ein. Bei Bedarf verordne ich aber auch Kräuter zum Einnehmen. Dabei fange ich mit Tropfen aus Kräuterkonzentraten an. Wenn diese nichts nützen, gehe ich zu Rohdrogen über. Natürlich muss die Dosis dem Körpergewicht angepasst werden.

Gessner: In der Homöopathie machen wir keinen prinzipiellen Unterschied zwischen der Behandlung von Kindern und Erwachsenen. Wir versu- chen immer, die Therapie individuell auf den Patienten auszurichten.

Viele Menschen empfinden die Alterung der Haut als Problem. Bietet die Ganzheitsmedizin Alternativen zu Botox und Lifting?

Thanh Huynh: Anti-Aging ist nicht mein Spezialgebiet. Ich denke aber, dass das beste Anti-Aging der Sonnenschutz ist. In der chinesischen Kräuterheilkunde gibt es Substanzen, die die Haut befeuchten, nähren und vitaler machen. Diese setze ich natürlich bei sehr trockener und rissiger Haut ein.

Gessner: Dass die alte Haut frisch bleibt, hängt mit verschiedenen Faktoren zusammen. Es kommt auf die Ernährung und die Lebensführung an. Viel Obst, Gemüse und wenig Alkohol helfen. Die entscheidende Frage ist, wie man als Mensch selbst reagiert, wenn die Haut faltig wird. Man kann sicherlich durch Ernährung, viel frische Luft und Sonnenschutz den Zustand der Haut positiv unterstützen. Es ist aber entscheidend, ob man als Mensch die Veränderungen akzeptiert. Das ist eine Frage der Haltung, ob man sehr empfindlich ist, wenn man anfängt, älter auszusehen. Wenn das ein starkes psychisches Problem ist, dann ist es aus meiner Sicht auch tatsächlich behandlungsbedürftig. Dann könnte man auch einen homöopathischen Ansatz wählen, um herauszufinden, was in dem Leben los ist, und so versuchen, die Einstellung zu beeinflussen. Die Homöopathie kann so zu größerer Gelassenheit beitragen.

Interview: Irène Dietschi, Oliver Klaffke 